\title{
Investigations on the Structural and Mechanical Properties of Polyurethane Resins Based on Cu(II)phthalocyanines
}

\author{
Tamer E. Youssef, ${ }^{1,2}$ Hamad Al-Turaif, ${ }^{1}$ and AbdulAziz A. Wazzan ${ }^{1}$ \\ ${ }^{1}$ Chemical and Materials Engineering Department, Faculty of Engineering, King Abdulaziz University, P.O. Box 80204, \\ Jeddah 21589, Saudi Arabia \\ ${ }^{2}$ Applied Organic Chemistry Department, National Research Center, Dokki, Cairo 12622, Egypt
}

Correspondence should be addressed to Tamer E. Youssef; tamezzat@yahoo.com

Received 31 May 2015; Revised 8 September 2015; Accepted 15 September 2015

Academic Editor: Jin Huang

Copyright (C) 2015 Tamer E. Youssef et al. This is an open access article distributed under the Creative Commons Attribution License, which permits unrestricted use, distribution, and reproduction in any medium, provided the original work is properly cited.

\begin{abstract}
This work report was reported on the effect of the addition of organic filler, that is, 2(3),9(10),16(17),23(24)-octahydroxycopper(II)phthalocyanine $\left[(\mathrm{OH})_{8} \mathrm{CuPc}\right](3)$, on the thermal, tensile, and morphological properties of a polyurethane matrix. The mechanical and dynamic mechanical thermal tests together with microstructural characterization of CuPc/PU composites were performed. The three PU composite films containing up to 1, 15, and $30 \mathrm{wt} \%$ of CuPc have different behaviors in terms of their morphological issues, thermal properties, and tensile behavior in comparison with the PU film as the reference material. Very high elongations at break from $910 \%$ to $1230 \%$, as well as high tensile strengths, illustrate excellent ultimate tensile properties of the prepared samples. The best mechanical and thermomechanical properties were found for the sample filled with $30 \mathrm{wt} \%$ of CuPc.
\end{abstract}

\section{Introduction}

Polyurethane (PU) is widely used polymeric material as a kind of coating for different daily life applications, such as fiber, automobile, construction, and biomaterials [1]. It improves the appearance and lifespan for many different materials. On automobiles, PU coatings give improved color retention, high gloss, corrosion resistance, and improved scratch [2]. Different types of PU coatings are used in building floors, concrete supports, and steel trusses which are spray coated. They were used against environmental deterioration [3].

There are six different groups of PU coating types assigned by ASTM. Most of them are high solids and solventless PU coatings [4]. PU are family of engineering thermoplastic elastomers.

It possess a good cost/performance ratio. Different kinds of polyurethane based coatings have been developed and used in different industries due to their superior properties, such as flexibility at low temperature, high abrasion resistance, high impact and tensile strength, high transparency, excellent gloss, color retention, corrosion protection properties, and good weathering resistance $[5,6]$. However, polyurethane has some disadvantages, that is, low thermal resistance, low adhesion, and low mechanical and anticorrosive properties [7].

In recent years, researchers have tried to conquest these disadvantages through different ways. Addition of pigments to the coatings has been introduced as an effective way to obtain polyurethane based coatings with enhanced mechani$\mathrm{cal}$ and anticorrosion properties $[8,9]$. In this regard, the use of fillers in polymer matrix allows for further improving of mechanical behavior. The resulted composites have properties different than the properties of the bulk polymer matrix itself [10-12].

There are a large number of reports in the literature indicating that the mechanical properties of the polyurethane based coatings can be significantly improved through improving tensile strength and Young's modulus of the coating [13].

The chemical structure and properties of polyurethane are influenced by using the metal complex systems based on transition metal for their synthesis, where metal complexes have the ability to order the chains, as well as have an effect 
on the chemical properties, and can improve the mechanical and anticorrosion properties of the coatings of polyurethanes [14].

They understood that the procedure of composite preparation is so important which can affect the filler dispersibility in the resin. It was shown that there is a logical relationship between the glass transition temperature $\left(T_{\mathrm{g}}\right)$ of the coating and the amount of resin chemically bonded to the filler surface. Fillers could increase the $T_{\mathrm{g}}$ of the coating when the resin segment chemically linked to the surface of particles. Depending on the surface nature of the filler, they could migrate to the surface of the coating or remain in the bulk $[15,16]$.

Metallophthalocyanines are interesting classes of organic fillers; they have been used as a new brand of materials to reach this target, due to their high specific surface area and thermal and chemical stability [17].

The contribution of our group to this field of research has been quite remarkable and described in detail in $[18,19]$.

As reported in literature, a considerable academic research efforts have described some metallophthalocyanine derivatives modified coatings for improving the mechanical and anticorrosion properties of the coatings. Yagi et al. [20] used cobalt(II)phthalocyanine as filler linked to a thermoplastic polyurethane resin.

In addition, copper phthalocyanine was used as a filler in a PU matrix to enhance its dielectric properties [21]. The mechanical and dynamic mechanical properties of Feoctacarboxyl acid phthalocyanine (Fe-OCAP)/PU was evaluated by tensile tests and dynamic mechanical analysis. The results indicated that the incorporation of Fe-OCAP significantly improved the tensile strength, elongation at break, and thermal deformation property of PU matrix [22].

Therefore, the improvements of the mechanical properties of polyurethane as mentioned above are essential for its industrial, technical requirements for several applications and help to create high performance PU coatings [23]. In light of the above considerations, this work reports how the presence of $\mathrm{CuPc}$ as filler can influence the mechanical behavior of polyurethane coating in composite forms based on bonding between $\mathrm{CuPc}$ and $\mathrm{PU}$ chains (referred to as $\mathrm{CuPc} / \mathrm{PU}$ here).

\section{Materials and Methods}

2.1. Materials. PU coating sample (Nitocoat UR512) was developed by Fosam Company Limited (Saudi Fosroc), Industrial Zone, Jeddah, KSA.

The octahydroxycopper(II)phthalocyanine (3) was synthesized as reported in our previous work [24]. Analytical grade dimethylformamide (DMF) was dried with $\mathrm{CaH}_{2}$, distilled in vacuo, and stored under dry nitrogen. The other reagents, such as organic solvents and bases, were analytical grade. Dimethylacetamide (DMAc) was purchased from Sigma Aldrich and used as received.

2.2. Apparatus. Microwave oven utilized for heating was used: Discover Lab Mate single mode microwave cavity from
CEM Corporation. The reactions were conducted in a $25 \mathrm{~mL}$ Schlenk tube, with a maximum operating temperature of $180^{\circ} \mathrm{C}$ and a maximum operating pressure of 8 bar.

2.3. Synthesis of 2(3),9(10),16(17),23(24)-Octahydroxycopper(II)phthalocyanine $\left[(\mathrm{OH})_{8} \mathrm{CuPc}\right](3)$. A solution of 2 was suspended in dichloromethane $(80 \mathrm{~mL})$ and $\mathrm{BBr}_{3}(20 \mathrm{~mL}$, $240 \mathrm{mmol}$ ) was added under $\mathrm{N}_{2}$. The mixture was stirred for 2 days at room temperature, then methanol was added slowly, and dark green suspensions were formed. The suspended solution was centrifuged. The precipitated solids were filtered off, washed with methanol, and dried under vacuum, to yield $80 \%$ of 3 , respectively, as black blue powder (Scheme 1).

IR (KBr) m ( $\left.\mathrm{cm}^{-1}\right)$ : 3308 (br, OH), 2953, 2925, 2851, 1660, 1542 (s), 1469 (s), 1440 (m), 1322 (m), 1274 (m), 1144 (s), 1130, $920(\mathrm{~s}), 880(\mathrm{~m})$, and $753(\mathrm{~m})$. UV-Vis (DMF), $\lambda_{\max }(\mathrm{nm}): 679$, 610, and 353. MS (FD): $m / z 704.07\left(\mathrm{M}^{+}\right)$.

2.4. Preparation of Copper Phthalocyanine/Polyurethane Composites. A solution-cast method was used to fabricate the $\mathrm{CuPc} / \mathrm{PU}$ composite films. A calculated amount of CuPc was added to a solution of PU in DMAC. The Ultra-Turrax T25 digital Homogenizer was used to complete blending the $\mathrm{CuPc}$ with the polyurethane/DMAC solution at high speed rotation $24.000 \mathrm{rpm}$ for 15 minutes. The mixture was then ultrasonically stirred for approximately $2 \mathrm{~h}$ to obtain a homogeneous solution. Afterwards, the mixture was casted on either glass or cement substrates at different wet thicknesses. The $\mathrm{CuPc} / \mathrm{Pu}$ films were left for 6 hours at room temperature, allowing for evaporation of the solvent. The schematic of possible dispersions of $\mathrm{CuPc}$ in $\mathrm{PU}$ was presented in Scheme 2. In this manner, three samples with $\mathrm{CuPc}$ content of 1,15 , and $30 \mathrm{wt} \%$ were prepared. The resulting composites were labeled $\mathrm{CuPc}(1 \%) / \mathrm{PU}, \mathrm{CuPc}(15 \%) / \mathrm{PU}$, and $\mathrm{CuPc}(30 \%) / \mathrm{PU}$, respectively.

2.5. Preparation of Test Specimens. Polymer specimens of $\mathrm{PU}$ or polymer composites of $\mathrm{CuPc} / \mathrm{PU}$ ca. $2 \mathrm{~mm}$ thickness were obtained by pouring the above mixture into the boneshaped molds. The molds were left at room temperature for 6 hours. After evaporation of the solvent. The resulting polymer composite specimens were then easily removed from the bone-shaped molds. They were stored under vacuum at room temperature until needed for testing.

2.6. Characterization Techniques. FT-IR spectra were recorded on a Bruker tensor 27 FT-IR spectrophotometer utilizing a Spectra-Tech ATR attachment model 0012-405 using a horizontal ZnSe crystal. Spectra were analyzed using Opus 6.0 software. The parameters used for the determination of each spectrum were the following: Mirror velocity: $0.4 \mathrm{~cm} / \mathrm{s}$ Spectral range: $400-4000 \mathrm{~cm}^{-1}$. Resolution: $8 \mathrm{~cm}^{-1}$ Number of scans: 512. The Ultraviolet-Visible Spectroscopy (UV-Vis) spectra of the fresh and produced $\mathrm{CuPc}$ were taken in $N, N$-dimethylformamide (DMF) using a Shimadzu UV-1800 spectrophotometer. FD mass spectra measurements were carried out with a Varian MAT 711 A spectrometer and 


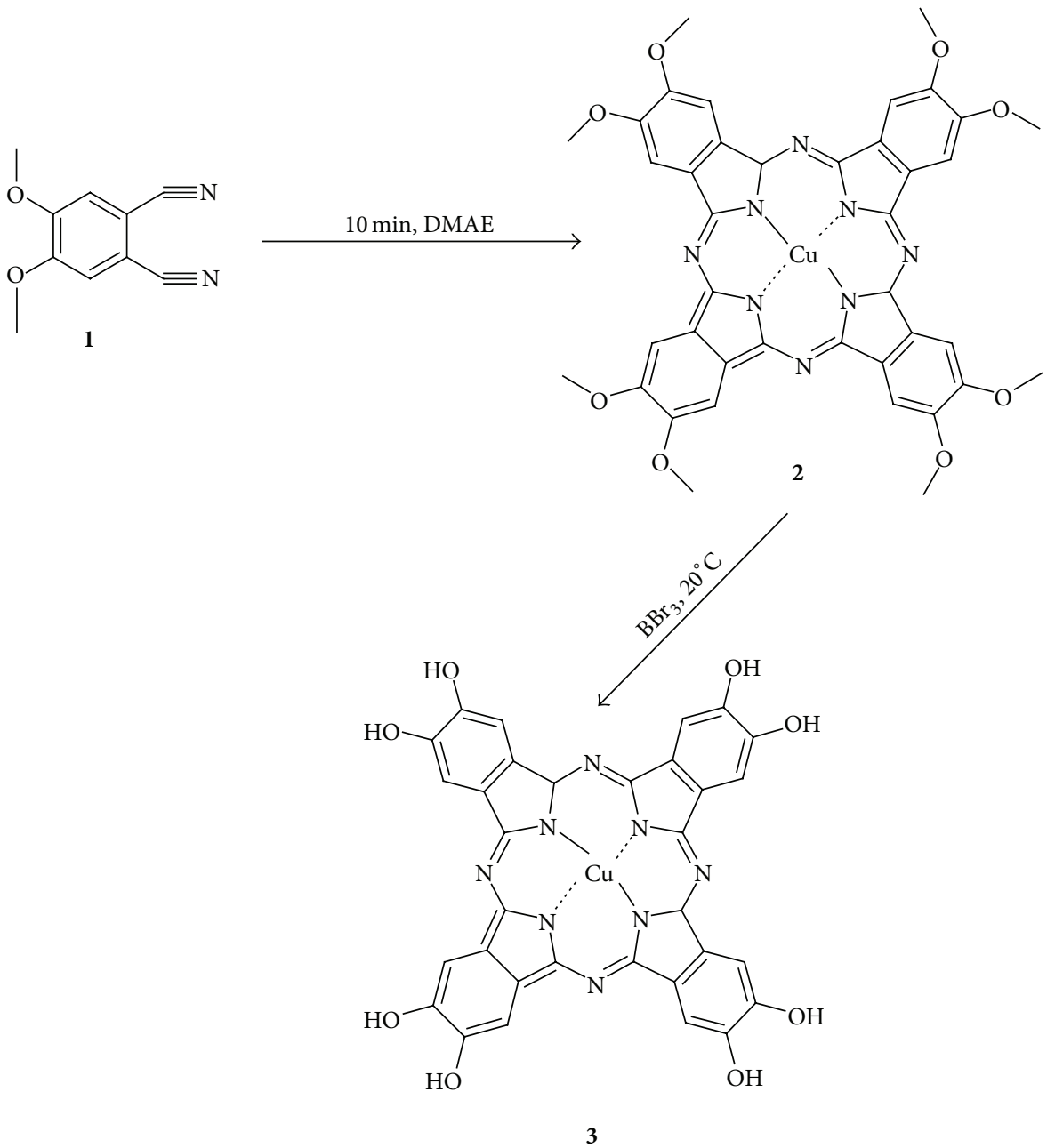

SCHEME 1: Preparation of octahydroxycopper(II)phthalocyanine 3.

reported as mass/charge $(\mathrm{m} / z)$. Elementary analyses were performed on a Carlo Erba Elemental Analyzer 1104, 1106.

XRD was performed on an INEL CPS 180 powder diffractometer EQUINOX 1000 system (filtered $\mathrm{Cu}$ Kal radiation, $30 \mathrm{KV} 30 \mathrm{~mA}$, spinning sample holder). The powder pattern analysis was processed by using Match software for phase identification with both COD and ICDD database, IMADINEL data processing software for graphical illustrations, MAUD software for Rietveld analysis method, and Fityk software for FWHM estimations.

Scanning electronic microscope (SEM) was used for investigating the surface morphology of $\mathrm{CuPc}, \mathrm{PU}$, and $\mathrm{CuPc} / \mathrm{PU}$. They were examined with a scanning electron microscope (JSM-6360LA, JEOL, Tokyo, Japan).

The abrasion resistance test of the films was measured using a Taber Abrasion Tester Model 5150. The pull-off test of the films was measured using an Elcometer adhesion tester. The test is done by securing loading fixtures (dollies) perpendicular to the surface of a coating with an adhesive.

The stress-strain behavior of the films was measured using an Instron Model 4400 Universal Testing System controlled by Series IX software. This type of machine has two crossheads; one is adjusted for the length of the specimen and the other is driven to apply tension to the test specimen.

Dynamic mechanical analyses were performed with a TA Q800 dynamic mechanical analyzer. The size of samples was about $11.0 \mathrm{~mm} \times 7.0 \mathrm{~mm} \times 0.30 \mathrm{~mm}$. A temperature scan mode was used and the temperature was ramped at a rate of $3^{\circ} \mathrm{C} / \mathrm{min}$ from -50 to $250^{\circ} \mathrm{C}$ under a nitrogen atmosphere with the frequency of $30 \mathrm{~Hz}$ and amplitude dynamic control of $15 \mu \mathrm{m}$ with dynamic forces of $100 \mathrm{mN}$. The parameters such as storage modulus, $\tan \delta$, and displacement as a function of temperature (which corresponded to the irreversible deformation) of the samples were obtained.

\section{Results and Discussion}

3.1. Characterization of Octahydroxycopper(II)phthalocyanine $\left[(\mathrm{OH})_{8} \mathrm{CuPc}\right](3)$. The first main task for this work was the synthetic route to novel peripherally octasubstituted copper(II)phthalocyanine 3, Scheme 1. It was synthesized as reported in our previous work [24]. The new copper phthalocyanine 3 was purified. 


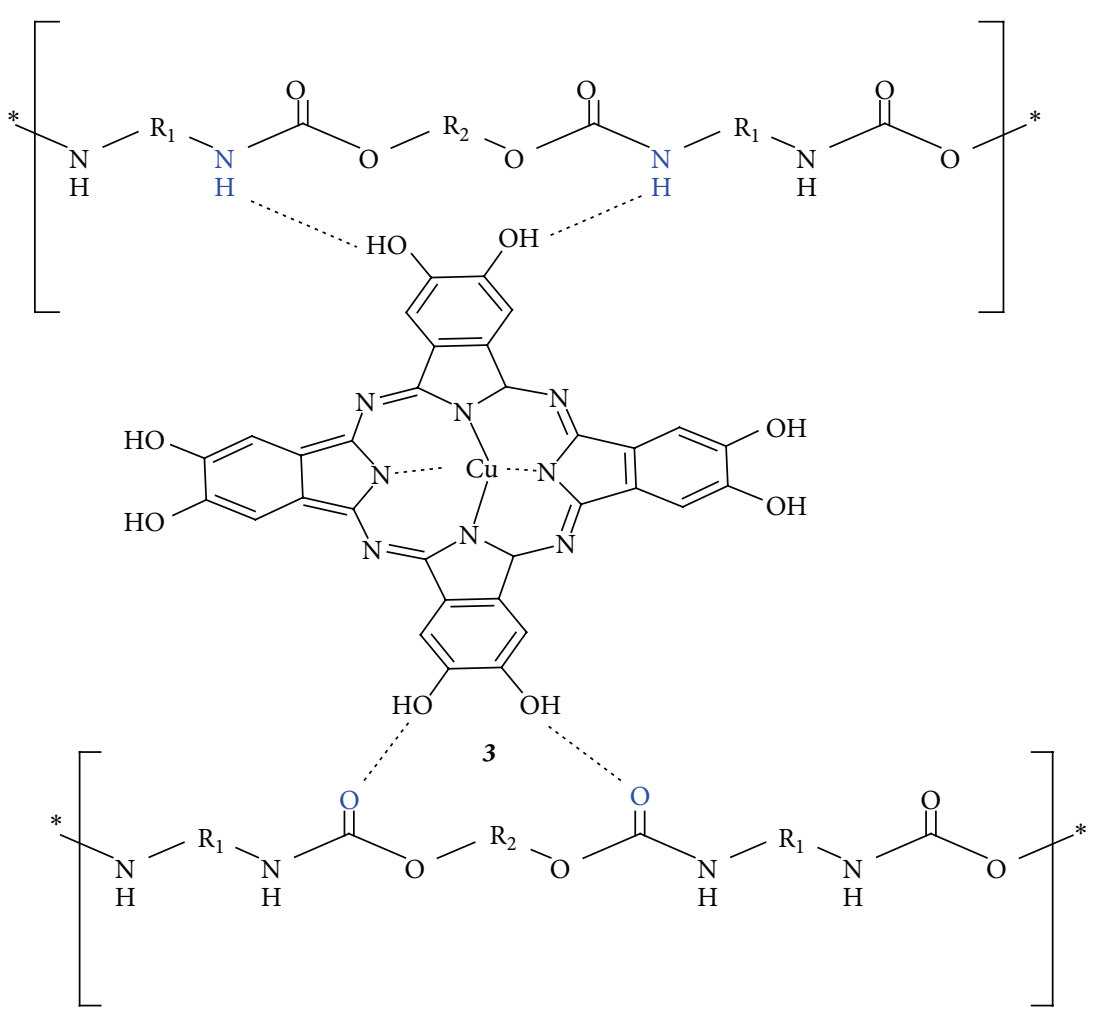

SCHEME 2: The schematic of possible dispersions of CuPc in PU skeleton.

It was characterized by spectral data (IR, UV-Visible spectroscopy, mass spectral data, and elemental analysis). The characterization data of the new compound were consistent with the assigned formula. In the IR spectra of octahydroxyphthalocyanine $\left[(\mathrm{OH})_{8} \mathrm{CuPc}\right](3)$, the appearance of $\mathrm{OH}$ stretched at $v=3228-3210 \mathrm{~cm}^{-1}$ was observed.

In the mass spectrum of $\mathbf{3}$, the presence of molecular ion peaks at $\mathrm{m} / z$ 704.07 $\left(\mathrm{M}^{+}\right)$confirmed the proposed structure. The result of elemental analysis also confirmed the structure of complex 3 . The electronic absorption spectra of 3 were carried out in DMF at room temperature. The split $\mathrm{Q}$ bands appeared at 679 and $610 \mathrm{~nm}$, while the split B band remained at $353 \mathrm{~nm}$. The $\mathrm{CuPc} / \mathrm{PU}$ composites were prepared by solution-cast method.

\subsection{Characterization of $\mathrm{CuPc/PU}$ Composite Films}

\subsubsection{Structural Properties of Cured Coatings}

Fourier-Transform Infrared Spectroscopy. In the polyurethane $\mathrm{N}=\mathrm{H}$ and $\mathrm{C}=\mathrm{O}$ are the significant bonds, which represent the urethane and the carbonyl linkages. The carbonyl in the amide group (-NHCO-) appeared at $v=1643 \mathrm{~cm}^{-1}$. The $\mathrm{N}-$ $\mathrm{H}$ stretching vibration overlapped with the vibration of the $\mathrm{O}-\mathrm{H}$ of the carboxyl group in CuPc appeared approximately at $\nu=3404-3435 \mathrm{~cm}^{-1}$. The $\mathrm{C}=\mathrm{O}$ stretching vibration (free urethane $\mathrm{C}=\mathrm{O}$ ) appeared at $\nu=1722 \mathrm{~cm}^{-1}$ and the absorption bands in the range of $925-933 \mathrm{~cm}^{-1}$ were attributed to the stretching vibration of $-\mathrm{C}-\mathrm{O}-\mathrm{C}-$.
$X$-Ray Diffraction $(X R D)$. The X-ray diffraction (XRD) patterns of pure $\mathrm{PU}, 1 \mathrm{wt} \%, 15 \mathrm{wt} \%$, and $30 \mathrm{wt} \%$ of $\mathrm{CuPc} / \mathrm{PU}$ were performed. The hard segments in the PU appeared at the diffraction peak near $2 \theta=20.26^{\circ}$, and the sharp peak were seen at $2 \theta$ value of $110.20^{\circ}$ indicative of metallic copper. The intensity of the peak at $2 \theta=73^{\circ}$ for $\mathrm{CuPc}(30 \%) / \mathrm{PU}$ was much stronger in comparison with that of $\mathrm{CuPc}(1 \%) / \mathrm{PU}$.

Energy-Dispersive X-Ray Spectroscopy (EDAX). The EDAX copper mapping for $\mathrm{PU}, 1 \mathrm{wt} \%, 15 \mathrm{wt} \%$, and $30 \mathrm{wt} \%$ of $\mathrm{CuPc} / \mathrm{PU}$ samples, respectively are shown in Figure 1. The pure PU clearly described a surface with no $\mathrm{Cu}$, whereas the other three surfaces contained a certain percentage of $\mathrm{Cu}$. The $1 \mathrm{wt} \% \mathrm{CuPc} / \mathrm{PU}$ composite sample showed an absorbance at $8.10 \mathrm{keV}$, but the $15 \mathrm{wt} \% \mathrm{CuPc} / \mathrm{PU}$ and $30 \mathrm{wt} \% \mathrm{CuPc} / \mathrm{PU}$ composite samples showed much larger absorbance's counts.

3.2.2. Optical and Imaging Properties of Cured Coatings. In order to examine the microstructure of the polyurethane composite coating samples, SEM analysis was conducted. Figures 2(a)-2(d) showed the SEM micrographs of the agglomerated crystal structure of $\mathrm{PU}$ and $\mathrm{CuPc} / \mathrm{PU}$ composites.

SEM micrographs of $\mathrm{PU}, \mathrm{CuPc}(1 \%) / \mathrm{PU}, \mathrm{CuPc}(15 \%) / \mathrm{PU}$, and $\mathrm{CuPc}(30 \%) / \mathrm{PU}$, respectively, were illustrated in Figure 2. It indicated the full dispersion of $\mathrm{CuPc}$ into $\mathrm{PU}$ chains. It was found that $\mathrm{CuPc}$ molecules aggregated into nearly spherical particles in the PU matrix for $\mathrm{CuPc}(1 \%) / \mathrm{PU}$, due to insufficient addition of $\mathrm{CuPc}$ amount; see Figure 2(b). This behavior was due to the planar shape and aromatic nature of CuPc. 


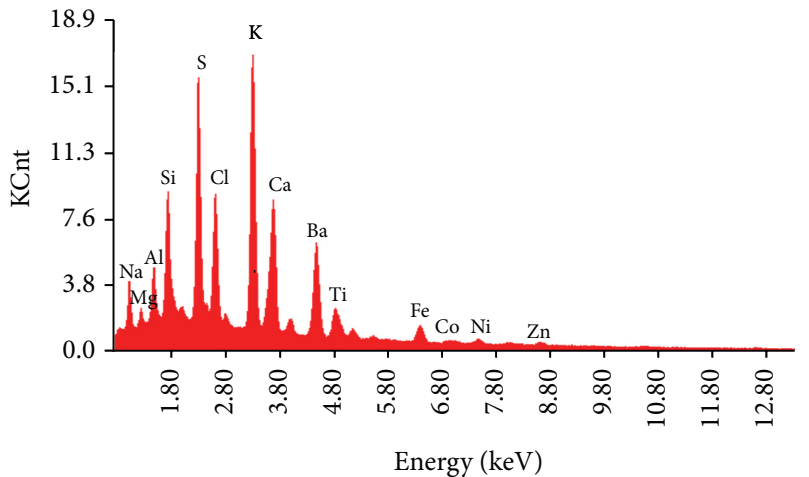

(a)

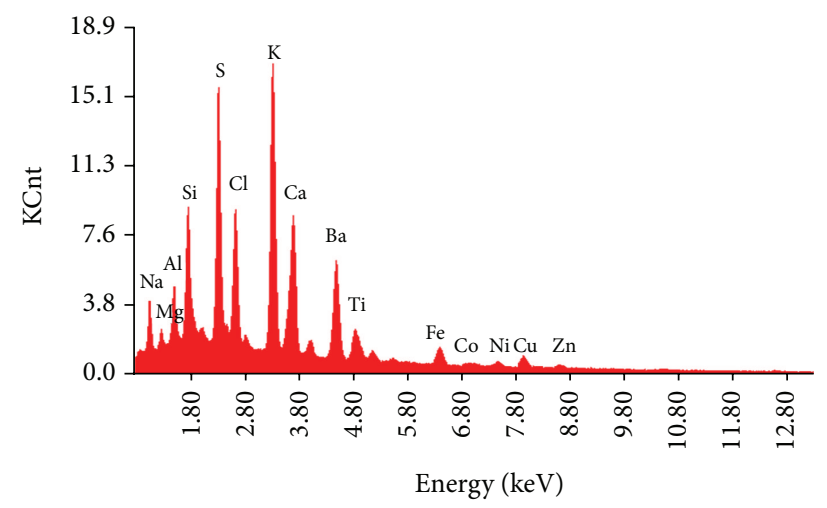

(c)

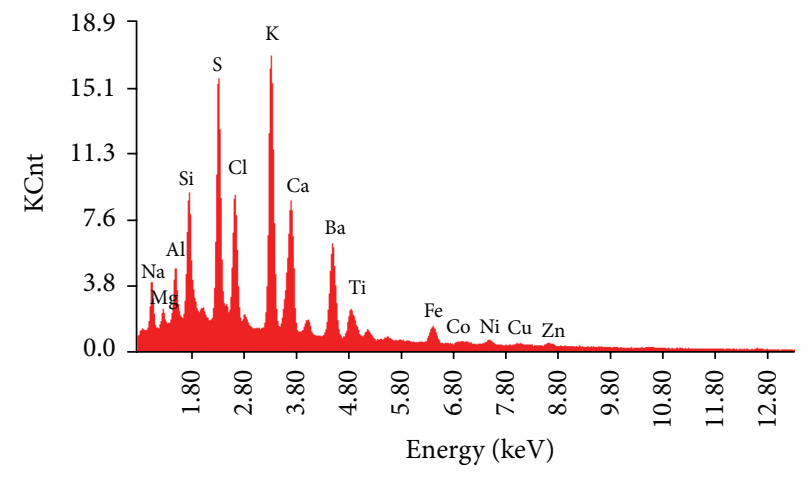

(b)

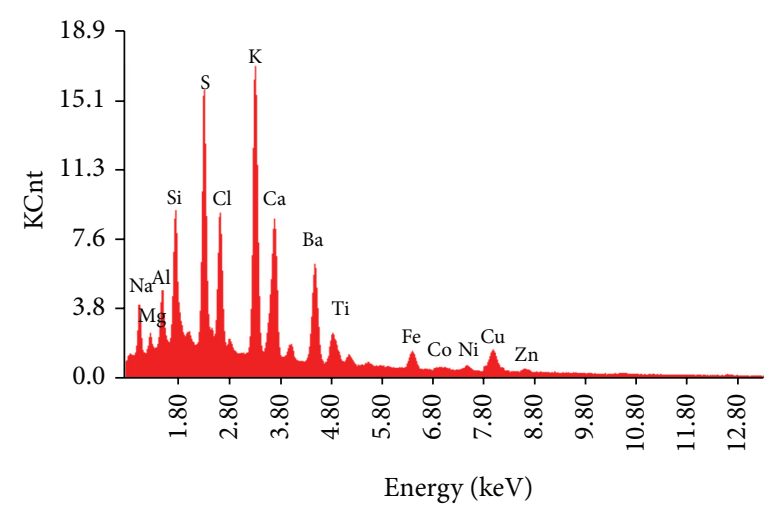

(d)

Figure 1: EDAX for PU (a), $\mathrm{CuPc}(1 \%) / \mathrm{PU}(\mathrm{b}), \mathrm{CuPc}(15 \%) / \mathrm{PU}(\mathrm{c})$, and $\mathrm{CuPc}(30 \%) / \mathrm{PU}(\mathrm{d})$, respectively.

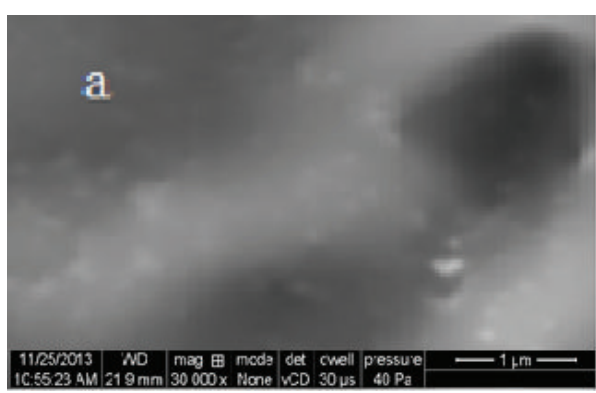

(a)

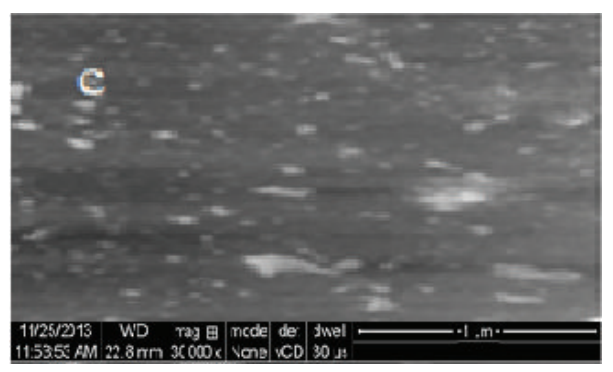

(c)

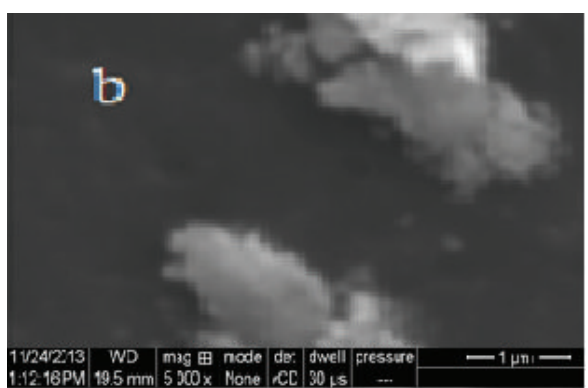

(b)

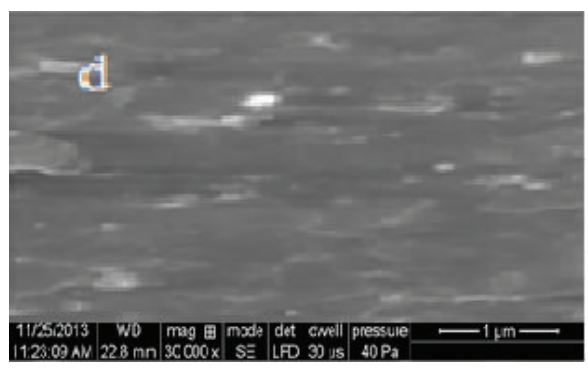

(d)

Figure 2: SEM Images of PU (a), CuPc(1\%)/PU (b), $\mathrm{CuPc}(15 \%) / \mathrm{PU}(\mathrm{c})$, and $\mathrm{CuPc}(30 \%) / \mathrm{PU}(\mathrm{d})$, respectively. 
TABLE 1: Abrasion test results for CuPc/PU composites.

\begin{tabular}{lcccc}
\hline Number of cycles & $\mathrm{PU}$ & $\mathrm{CuPc}(1 \%) / \mathrm{PU}$ & $\mathrm{CuPc}(15 \%) / \mathrm{PU}$ & $\mathrm{CuPc}(30 \%) / \mathrm{PU}$ \\
\hline 100 cycles & 0.08 & 0.05 & 0.04 & 0.03 \\
200 cycles & 0.03 & 0.01 & 0.01 & 0.01 \\
300 cycles & 0.03 & 0.01 & 0.02 & 0.02 \\
400 cycles & 0.04 & 0.03 & 0.02 & 0.02 \\
500 cycles & 0.03 & 0.02 & 0.11 & 0.02 \\
Total weight loss in gm & 0.21 & 0.12 & 0.10 \\
\hline
\end{tabular}

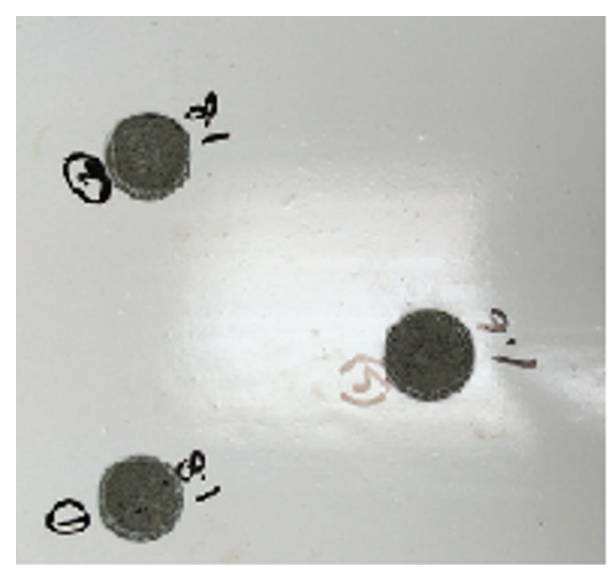

(a)

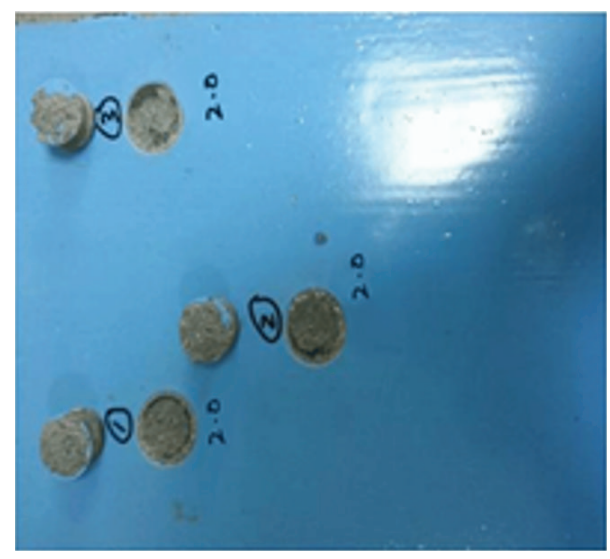

(c)

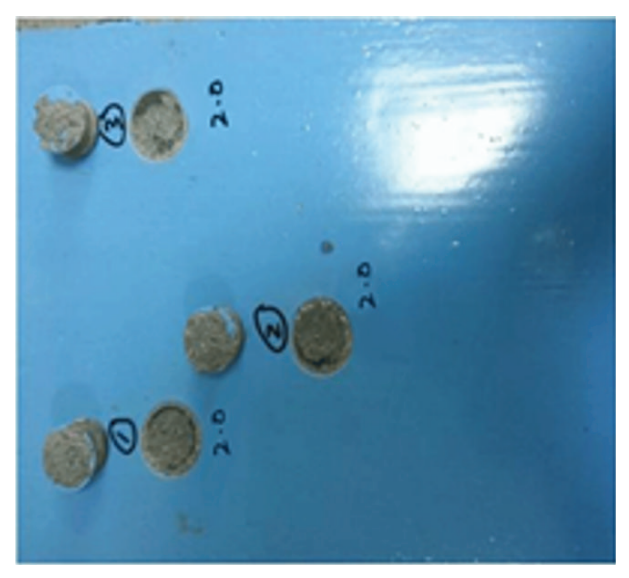

(b)

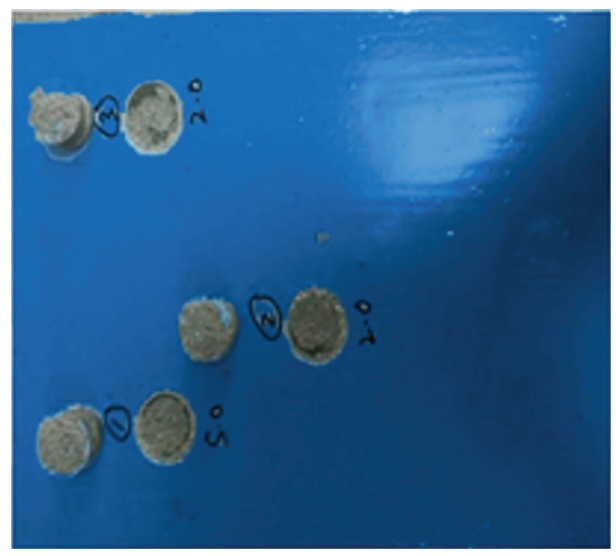

(d)

Figure 3: Adhesion test of PU (a), CuPc(1\%)/PU (b), CuPc(15\%)/PU (c), and $\mathrm{CuPc}(30 \%) / \mathrm{PU}(\mathrm{d})$, respectively.

The aggregation behavior was smaller than in the case of the particles in $\mathrm{CuPc}(15 \%) / \mathrm{PU}$ sample; confer Figure 2(c). It can be observed that the mobility of the bonded $\mathrm{CuPc}$ was hindered by the PU chains and the growth of $\mathrm{CuPc}$ crystals was limited. Actually, the aggregation of $\mathrm{CuPc}$ readily occurred in $\mathrm{CuPc}(1 \%) / \mathrm{PU}$. The shapes of the $\mathrm{CuPc}$ particulates change into irregular shape. It showed improved separation and decreased agglomeration compared to that of PU sample.

\subsubsection{Mechanical Properties}

Abrasion Test. Resistance to abrasion of the surface of PU composite samples was established by differential weighing of the specimen through a specific number of test cycles. Poor abrasion was denoted by higher weight loss as in pure PU samples, while good abrasion was denoted by less weight loss as in $\mathrm{CuPc} / \mathrm{PU}$ composite samples as shown in Table 1.

Adhesion Test. The adherent strength of the PU sample and cured films of $\mathrm{CuPc} / \mathrm{PU}$ were shown in Figure 3. All $\mathrm{CuPc} / \mathrm{PU}$ composite film samples exhibited good adhesion to mild concrete substrates; it was quite flexible, good impact resistance and hardness, except pure PU sample failed in impacting resistance and flexibility, where the record value of adhesion for PU was 1.73 MPa and was raised to $3.00 \mathrm{MPa}$ for $\mathrm{CuPc} / \mathrm{PU}$ samples. 


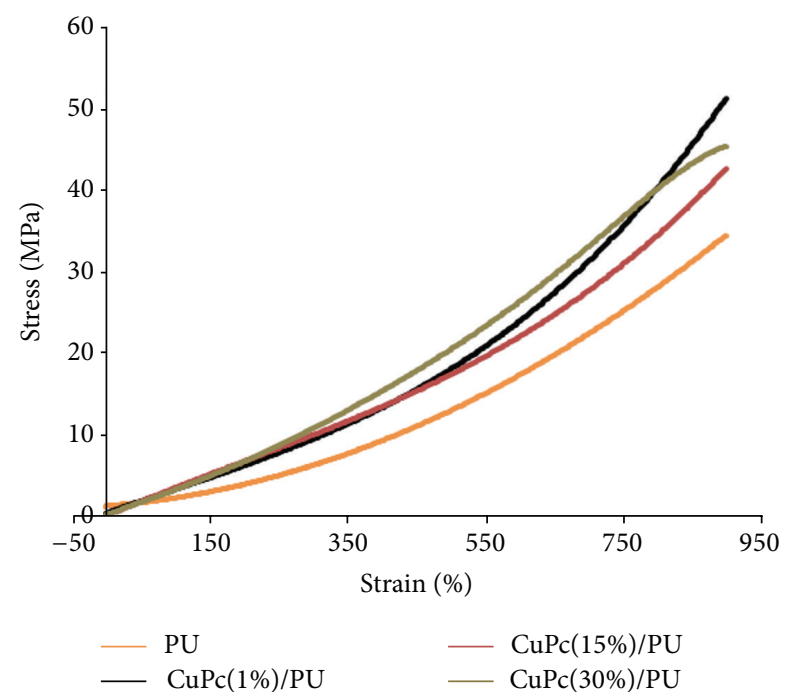

FIgURE 4: Stress-Strain behavior for PU and CuPc/PU composites.

Stress-Strain Analysis. The stress-strain tests for $\mathrm{CuPc} / \mathrm{PU}$ composite films were carried out to understand the effect of $\mathrm{CuPc}$ reinforcement on the physical and mechanical properties of the PU composite films, when a load transfers from PU to $\mathrm{CuPc}$.

The stress-strain curves of pure $\mathrm{PU}$ and the $\mathrm{CuPc} / \mathrm{PU}$ composite films with different ratio contents of $\mathrm{CuPc}$ are shown in Figure 4. The tensile strength of the samples increased from 31.1 to $58.3 \mathrm{MPa}$; also the elongation at break of the samples increased from $910 \%$ to $1230 \%$ due to the increasing of $\mathrm{CuPc}$ content from 1 to $30 \%$. The rapid increase in tensile strength and the elongation of the uncrosslinked polyurethane phase happened through the stress-strain relationship. In addition, the $\mathrm{CuPc} / \mathrm{PU}$ composite film showed a uniform change, as evidence for a more bounded network. This behavior was close to the ideal (theoretical) stress-strain curve as shown in Figure 4.

The elastomeric behavior of $\mathrm{CuPc} / \mathrm{PU}$ composites was observed; the values of Young's modulus, tensile strength, and elongation at the break point of the $\mathrm{CuPc} / \mathrm{PU}$ composite films were different than values of PU matrix.

The mechanical properties of $\mathrm{CuPc} / \mathrm{PU}$ composite films were more excellent than those of pure PU, due to the highly homogeneously dispersion of $\mathrm{CuPc}$ within the PU chains. Table 2 showed the corresponding tensile strength and the elongation at break of the samples.

3.2.4. Dynamic Mechanical Thermal Properties. The dynamic mechanical thermal analysis (DMTA) investigations for PU sample showed poor mechanical properties due to its more uniformly cross-linking for $\mathrm{CuPc}(1 \%) / \mathrm{PU}, \mathrm{CuPc}(15 \%) / \mathrm{PU}$, and $\mathrm{CuPc}(30 \%) / \mathrm{PU}$ samples exhibited different dynamic mechanical properties, and they have the best mechanical properties.

Similarly, DMTA can reveal significant details about the structure property relationships of PU films. Viscoelastic properties such as storage modulus, loss modulus, and $\tan \delta$
TABLE 2: Mechanical properties of PU and CuPc/PU composites.

\begin{tabular}{lcc}
\hline Sample & Tensile strength (MPa) & Elongation at break (\%) \\
\hline $\mathrm{PU}$ & 31.1 & 910 \\
$\mathrm{CuPc}(1 \%) / \mathrm{PU}$ & 32.4 & 982 \\
$\mathrm{CuPc}(15 \%) / \mathrm{PU}$ & 40.8 & 990 \\
$\mathrm{CuPc}(30 \%) / \mathrm{PU}$ & 58.3 & 1230 \\
\hline
\end{tabular}

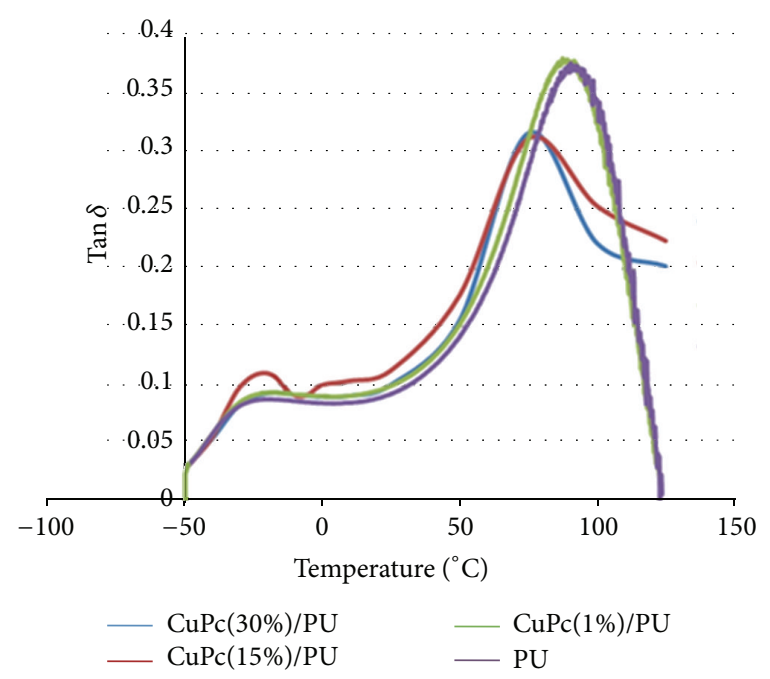

Figure 5: Temperature dependence of loss tangent $(\tan \delta)$ for the $\mathrm{PU}, \mathrm{CuPc}(1 \%) / \mathrm{PU}, \mathrm{CuPc}(15 \%) / \mathrm{PU}$, and $\mathrm{CuPc}(30 \%) / \mathrm{PU}$ samples.

were obtained as functions of time, frequency, and temperature. The DMTA experiments were done for our PU film samples to know the variation of the mechanical dynamic properties between them.

The curves of $\tan \delta$ as a function of temperature for PU and $\mathrm{CuPc} / \mathrm{PU}$ composite films showed a loss peak around $-25^{\circ} \mathrm{C}$, which corresponds to the $T_{\mathrm{g}}$ of soft segments in $\mathrm{PU}$ as shown in Figure 5. At the temperature above $50^{\circ} \mathrm{C}$, the $\tan \delta$ values of the samples increased corresponding to the $T_{\mathrm{g}}$ of hard segments. However, a clear irreversible deformation of samples was developed in the temperature range.

The logarithm of the storage modulus (E) versus temperature for the pure $\mathrm{PU}, \operatorname{CuPc}(1 \%) / \mathrm{PU}, \operatorname{CuPc}(15 \%) / \mathrm{PU}$, and $\mathrm{CuPc}(30 \%) / \mathrm{PU}$ was shown in Figure 6. The values of $\grave{\mathrm{E}}$ of the composite samples were below $-50^{\circ} \mathrm{C}$. When the temperature was above $-50^{\circ} \mathrm{C}$, the values of $\mathrm{E}$ increased with the increase of filler $\mathrm{CuPc}$ content, because the samples were in the glassy state below temperature of $-50^{\circ} \mathrm{C}$, which reduce the molecular motion of both hard and soft segments. At temperature above the $T_{\mathrm{g}}$ of soft segments, a broad plateau modulus appeared.

It can be seen from Figure 6 that the value of $\dot{E}$ of PU decreased quickly with increasing of temperature, while it decreased in the case of the $\mathrm{CuPc} / \mathrm{PU}$ composites. This result approved that the addition of filler $\mathrm{CuPc}$ enhanced the physical cross-links in PU matrix and improved the mechanical properties of composites. 


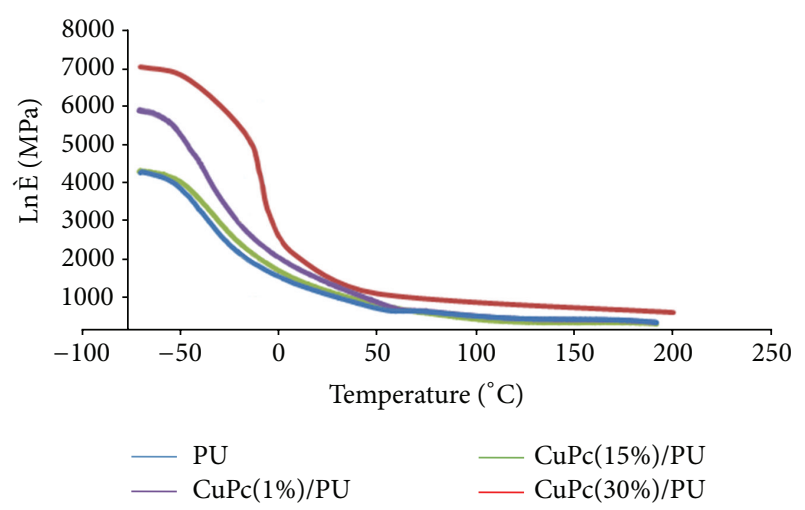

FIgUre 6: Storage modulus (亡̀) for the $\mathrm{PU}, \mathrm{CuPc}(1 \%) / \mathrm{PU}$, $\mathrm{CuPc}(15 \%) / \mathrm{PU}$, and $\mathrm{CuPc}(30 \%) / \mathrm{PU}$ samples.

The irreversible deformation of the $\mathrm{PU}$ and $\mathrm{CuPc} / \mathrm{PU}$ composite samples as a function of temperature was shown in Figure 7. The temperatures of deformation of $\mathrm{PU}$, $\mathrm{CuPc}(1 \%) / \mathrm{PU}, \mathrm{CuPc}(15 \%) / \mathrm{PU}$, and $\mathrm{CuPc}(30 \%) / \mathrm{PU}$ were $170^{\circ} \mathrm{C}, 175^{\circ} \mathrm{C}, 180^{\circ} \mathrm{C}$, and $190^{\circ} \mathrm{C}$, respectively. The thermal deformation was improved with increasing of the content of filler $\mathrm{CuPc}$.

\subsubsection{Chemical Properties of Cured Coatings}

UV Weathering Resistant. Coatings are chemically degraded by the effect of UV radiation depending on duration and intensity. In the case of pure PU,a surface embrittlement was formed, followed by a slightly yellowing in the color of the sample.

Addition of filler may improve UV resistance and prevent the deep penetration of UV rays and mechanical destruction. The modified PU with CuPc showed an improvement in the UV resistance. The CuPc/PU samples clearly indicate no degradation or surface discoloration of composite coated samples when exposed to UV radiation.

The surface micrographs of UV exposed for PU and $\mathrm{CuPc} / \mathrm{PU}$ samples after 1000 hours were shown in Figure 8.

Chemical Resistance Properties. The chemical resistance of PU and $\mathrm{CuPc} / \mathrm{PU}$ coating series in acid and base solutions was performed for the cured films. No changes were observed for the PU coating modified with $1 \%, 15 \%$, and $30 \% \mathrm{CuPc}$. No appearance of blue color of $\mathrm{Cu} / \mathrm{PU}$ composites impressed in the acid or alkaline solutions was clearly observed. The acid solutions colors were still transparent until thirty days of the experiment, whereas slightly blue color appeared in basic solutions from the thirty day; it may be due to the action of alkali on urea bonds in PU. This suggests that there is no chemical degradation of $\mathrm{Cu} / \mathrm{PU}$ composites and the performance of the coating was strong.

It was known that the chemical resistance depends on several factors like the concentration, the period of exposure, the temperature, and the quantity.

Contact of composite coating samples with saturated hydrocarbons, such as kerosene, results in a limited swelling.

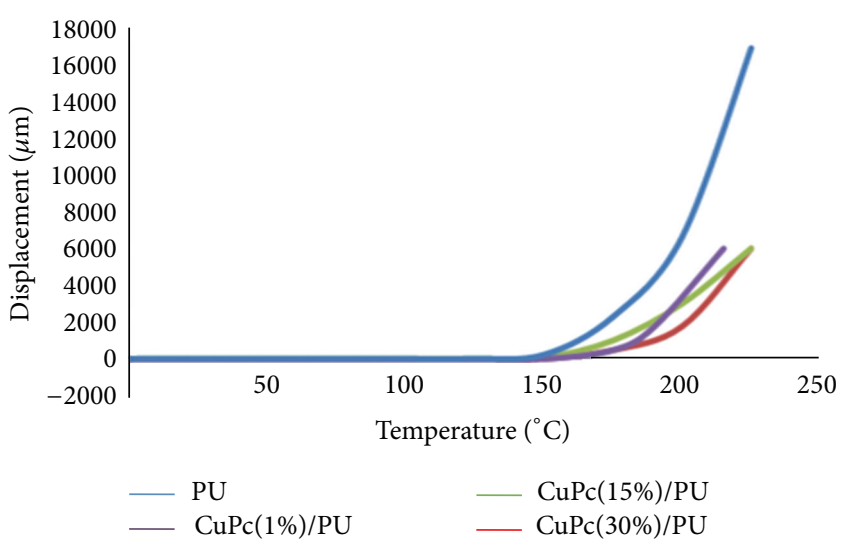

Figure 7: Irreversible deformation of $\mathrm{PU}, \mathrm{CuPc}(1 \%) / \mathrm{PU}$, $\mathrm{CuPc}(15 \%) / \mathrm{PU}$, and $\mathrm{CuPc}(30 \%) / \mathrm{PU}$ samples.

Contact of composite coating samples, such as toluene and benzene, afforded a considerable swelling even at room temperature. As ethanol, swelling of composite coating samples caused and lead to a loss of tensile strength.

Ketones such as acetone are partial solvents for thermoplastic polyurethane elastomers. Solvents like ethyl acetate cause swelling of composite coating samples. Solvents such as dimethylformamide (DMF), dimethyl sulfoxide (DMSO), and tetrahydrofuran (THF) dissolve all polyurethane coating samples.

\section{Conclusions}

Finally, we wish to comment on the state of our $\mathrm{CuPc}$ filler dye. Our results indicated a considerable improvements in mechanical and thermomechanical properties of PU.

As already mentioned above, in the formed composites, the size of the filler CuPc particles dispersed in the PU matrix has enhancement of the mechanical response of the formed composites.

On the other hand, the values of Young's and storage moduli increased with the increase of CuPc filler content, indicating good rigidity (storage and Young moduli) of the samples, high values of the mechanical parameters, and greater thermal stability, and could prove to be a very valuable industrially.

All CuPc/PU samples exhibited good adhesion to mild concrete substrates; it was quite flexible, good impact resistance and hardness, and the record value of adhesion for PU was $1.73 \mathrm{MPa}$ and raised to $3.00 \mathrm{MPa}$ for $\mathrm{CuPc} / \mathrm{PU}$ samples.

Poor abrasion was denoted by higher weight loss as in $\mathrm{PU}$ sample, while good abrasion was denoted by less weight loss as in $\mathrm{CuPc} / \mathrm{PU}$ sample. The weight loss of the PU specimen after 500 cycles was $0.21 \mathrm{~g}$ but for $\mathrm{CuPc} / \mathrm{PU}$ specimen was $0.10 \mathrm{~g}$ only.

The stress-strain curves of $\mathrm{PU}$ and the composites with different ratio contents of $\mathrm{CuPc}$ showed an increase in the tensile strength of $31.1 \mathrm{MPa}(\mathrm{PU})$ to $58.3 \mathrm{MPa}(\mathrm{CuPc} / \mathrm{PU})$ and the elongation at break of the samples increased from 910\% to $1230 \%$ for the PU and CuPc/PU composite samples, respectively. 


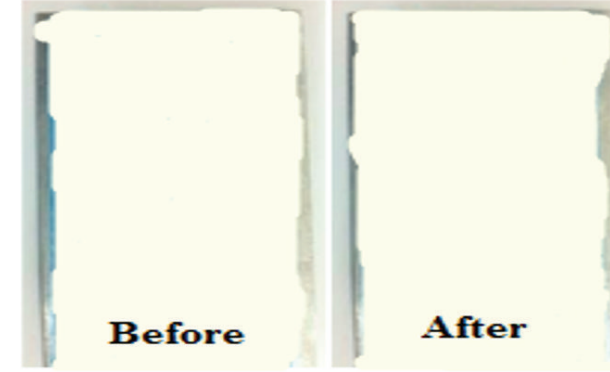

(a)

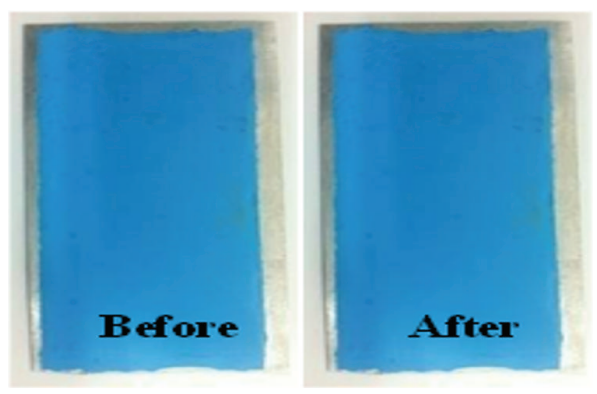

(c)

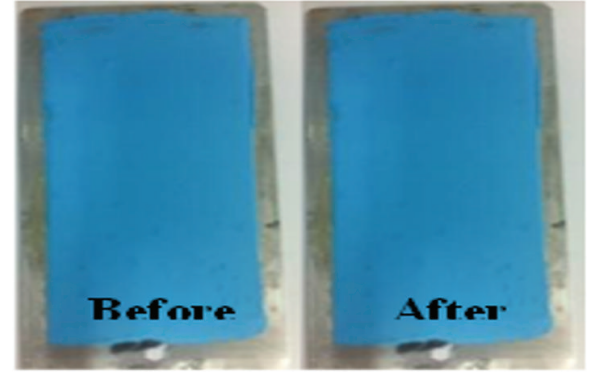

(b)

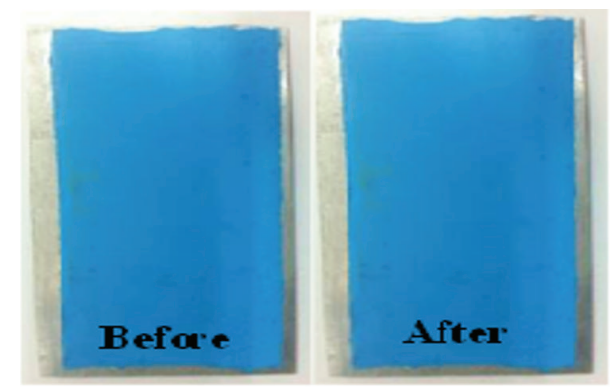

(d)

Figure 8: Samples of PU (a), $\mathrm{CuPc}(1 \%) / \mathrm{PU}(\mathrm{b}), \mathrm{CuPc}(15 \%) / \mathrm{PU}$ (c), and $\mathrm{CuPc}(30 \%) / \mathrm{PU}$ (d) before and after being exposed to UV.

\section{Conflict of Interests}

The authors declare that there is no conflict of interests regarding the publication of this paper.

\section{Acknowledgments}

This project was funded by Saudi Basic Industries Corporation (SABIC) and the Deanship of Scientific Research (DSR), King Abdulaziz University, Jeddah, under Grant no. (MS/15/236/1434). The authors, therefore, acknowledge with thanks SABIC and DSR technical and financial support.

\section{References}

[1] R. G. J. C. Heijkants, R. V. Van Calck, T. G. Van Tienen et al., "Uncatalyzed synthesis, thermal and mechanical properties of polyurethanes based on poly( $\varepsilon$-caprolactone) and 1,4-butane diisocyanate with uniform hard segment," Biomaterials, vol. 26, no. 20, pp. 4219-4228, 2005.

[2] C. Prisacariu, Polyurethane Elastomers from Morphology to Mechanical Aspects, Springer, New York, NY, USA, 2011.

[3] M. N. Radhakrishnan Nair and M. R. Gopinathan Nair, "Synthesis and characterisation of soluble block copolymers from NR and TDI based polyurethanes," Journal of Materials Science, vol. 43, no. 2, pp. 738-747, 2008.

[4] R. H. Brown, "Initial effects of clear coatings on water permeance of masonry," in Masonry: Materials, Properties, and Performance, J. G. Borchelt, Ed., ASTM STP 778, pp. 221-236, ASTM International, West Conshohocken, Pa, USA, 1982.

[5] M. I. Aranguren, "Polymer composites", in Encyclopedia of Surface and Colloid Science, P. Somasundaran and A. Hubbard,
Eds., vol. 6, pp. 4796-4810, Taylor \& Francis/CRC Press, New York, NY, USA, 2nd edition, 2006.

[6] S. A. Madbouly and A. Lendlein, "Shape-memory polymer composites in shape memory polymers," in Advances in Polymer Science, A. Lendlein, Ed., vol. 226, pp. 41-95, Springer, Berlin, Germany, 2010.

[7] U. M. Casado, N. E. Marcovich, M. I. Aranguren, and M. A. Mosiewicki, "High-strength composites based on tung oil polyurethane and wood flour: effect of the filler concentration on the mechanical properties," Polymer Engineering and Science, vol. 49, no. 4, pp. 713-721, 2009.

[8] M. Heidarian, M. R. Shishesaz, S. M. Kassiriha, and M. Nematollahi, "Characterization of structure and corrosion resistivity of polyurethane/organoclay nanocomposite coatings prepared through an ultrasonication assisted process," Progress in Organic Coatings, vol. 68, no. 3, pp. 180-188, 2010.

[9] S. Ashhari, A. A. Sarabi, S. M. Kasiriha, and D. Zaarei, "Aliphatic polyurethane-montmorillonite nanocomposite coatings: preparation, characterization, and anticorrosive properties," Journal of Applied Polymer Science, vol. 119, no. 1, pp. 523529, 2011.

[10] S. K. Dhoke, A. S. Khanna, and T. J. M. Sinha, "Effect of nano-ZnO particles on the corrosion behavior of alkyd-based waterborne coatings," Progress in Organic Coatings, vol. 64, no. 4, pp. 371-382, 2009.

[11] S. K. Dhoke and A. S. Khanna, "Effect of nano- $\mathrm{Fe}_{2} \mathrm{O}_{3}$ particles on the corrosion behavior of alkyd based waterborne coatings," Corrosion Science, vol. 51, no. 1, pp. 6-20, 2009.

[12] A. Aglan, A. Allie, A. Ludwick, and L. Koons, "Formulation and evaluation of nano-structured polymeric coatings for corrosion protection," Surface and Coatings Technology, vol. 202, no. 2, pp. 370-378, 2007. 
[13] H. J. Qi and M. C. Boyce, "Stress-strain behavior of thermoplastic polyurethanes," Mechanics of Materials, vol. 37, no. 8, pp. 817839, 2005.

[14] X. Yan and G. Xu, "Corrosion and mechanical properties of polyurethane/Al composite coatings with low infrared emissivity," Journal of Alloys and Compounds, vol. 491, no. 1-2, pp. 649653, 2010.

[15] Z.S. Petrović, I. Javni, and V. Divjaković, "Structure and physical properties of segmented polyurethane elastomers containing chemical crosslinks in the hard segment," Journal of Polymer Science B: Polymer Physics, vol. 36, no. 2, pp. 221-235, 1998.

[16] B. Hartmann, J. V. Duffy, G. F. Lee, and E. Balizer, "Thermal and dynamic mechanical properties of polyurethane," Journal of Applied Polymer Science, vol. 35, pp. 1829-1852, 1998.

[17] G. Löbbert, "Phthalocyanines," in Ullmann's Encyclopedia of Industrial Chemistry, Wiley-VCH, Weinheim, Germany, 2002.

[18] T. E. Youssef and A. M. Abdel Ghaffar, "Polystyrene/iron phthalocyanine composite: radiation effect and characterization," Polymer Composites, vol. 28, no. 5, pp. 631-636, 2007.

[19] T. E. Youssef and A. M. Abdel Ghaffar, "Preparation and radiation effect on composite films containing both polypropylene and novel phthalocyanines with functionalized bulky phenoxy groups," Journal of Applied Polymer Science, vol. 119, no. 1, pp. 134-141, 2011.

[20] S. Yagi, M. Kimura, T. Koyama, K. Hanabusa, and H. Shirai, "Functional metallomacrocycles and their polymers XXXIII. Autoxidation of thiol catalyzed by thermoelastic polyurethane resin containing a cobalt(II)phthalocyanine," Polymer Journal, vol. 27, no. 11, pp. 1139-1143, 1995.

[21] J. Wang, C. Wu, R. Liu, and S. Li, "Enhanced dielectric behavior in nanocomposites of polyurethane bonded with copper phthalocyanine oligomers," Polymer Journal, vol. 46, no. 5, pp. 285-292, 2014.

[22] Y. Ti and D. Chen, "Mechanical and dynamic mechanical properties of polyurethane/Fe-octacarboxyl acid phthalocyanine blends," Progress in Organic Coatings, vol. 76, no. 1, pp. 119124, 2013.

[23] I. M. Davletbaeva, V. F. Shkodich, A. I. Ismagilova, and V. V. Parfenov, "Electro-physical properties of mesogenic metalcoordinated polyurethanes," Russian Polymer News, vol. 6, pp. 36-38, 2011.

[24] T. E. Youssef, "Efficient green procedures for the preparation of novel tetraalkynyl-substituted phthalocyanines," Polyhedron, vol. 29, no. 7, pp. 1776-1783, 2010. 

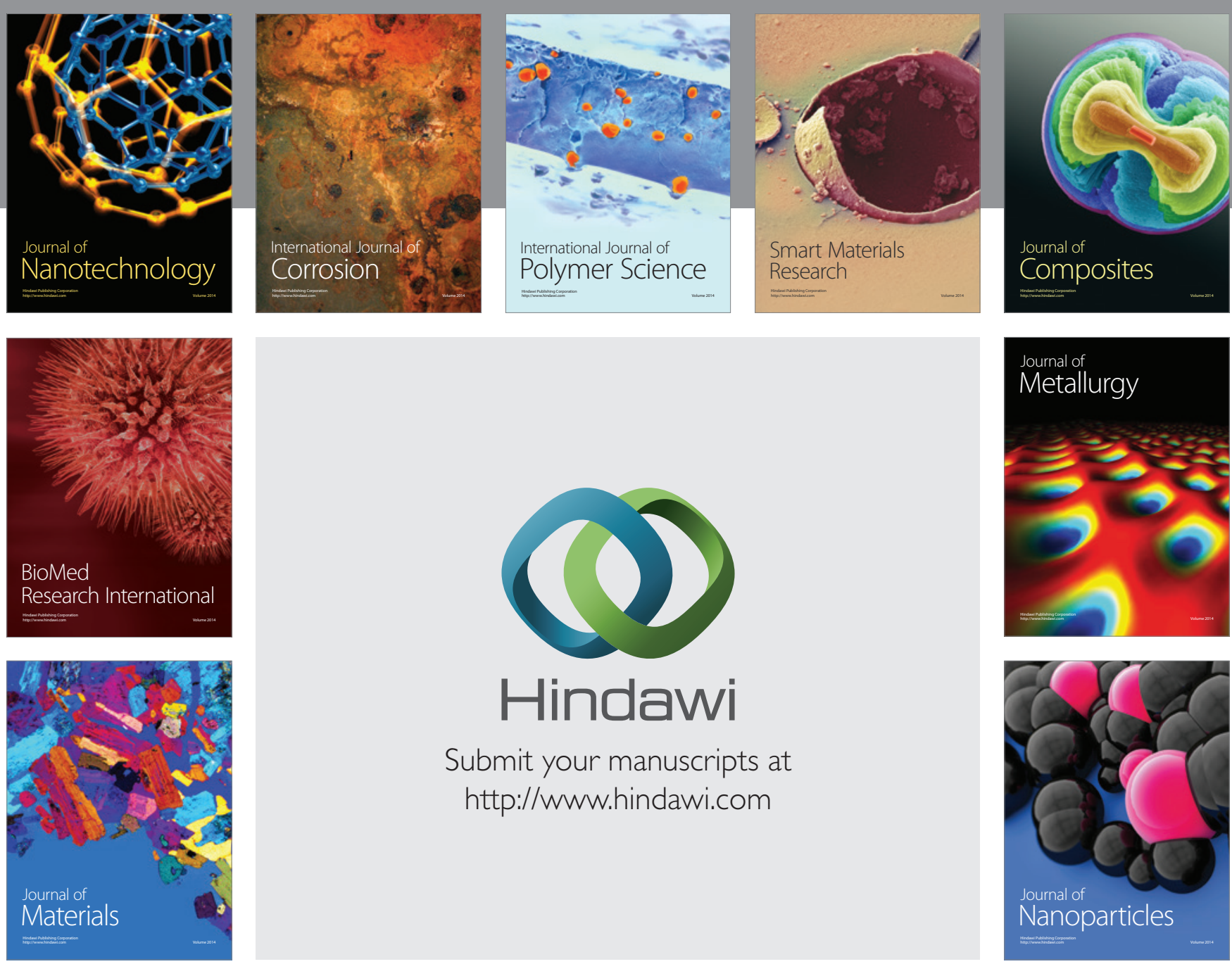

Submit your manuscripts at http://www.hindawi.com
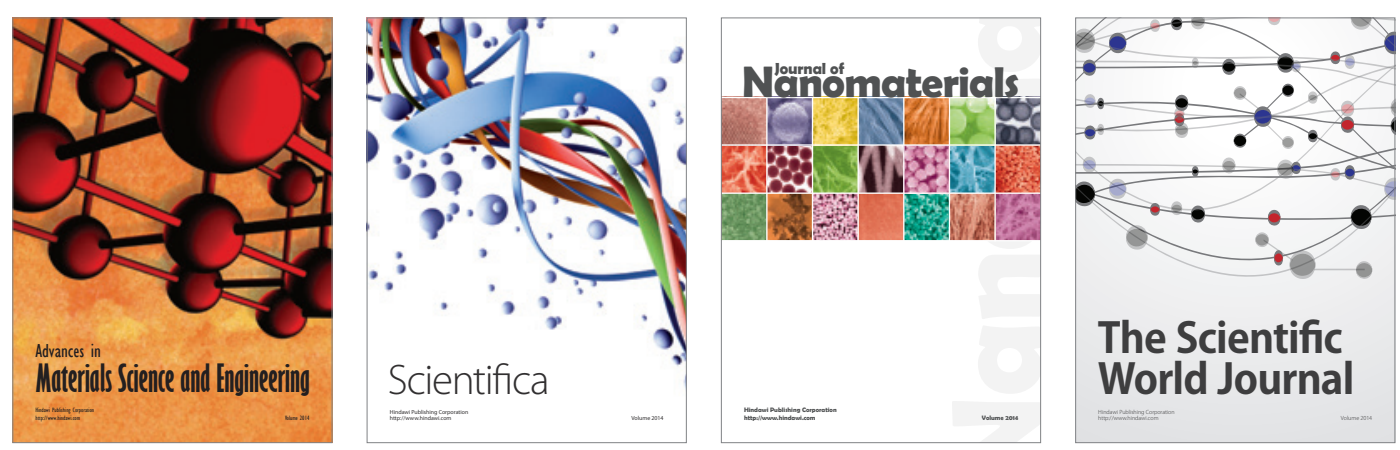

\section{The Scientific World Journal}
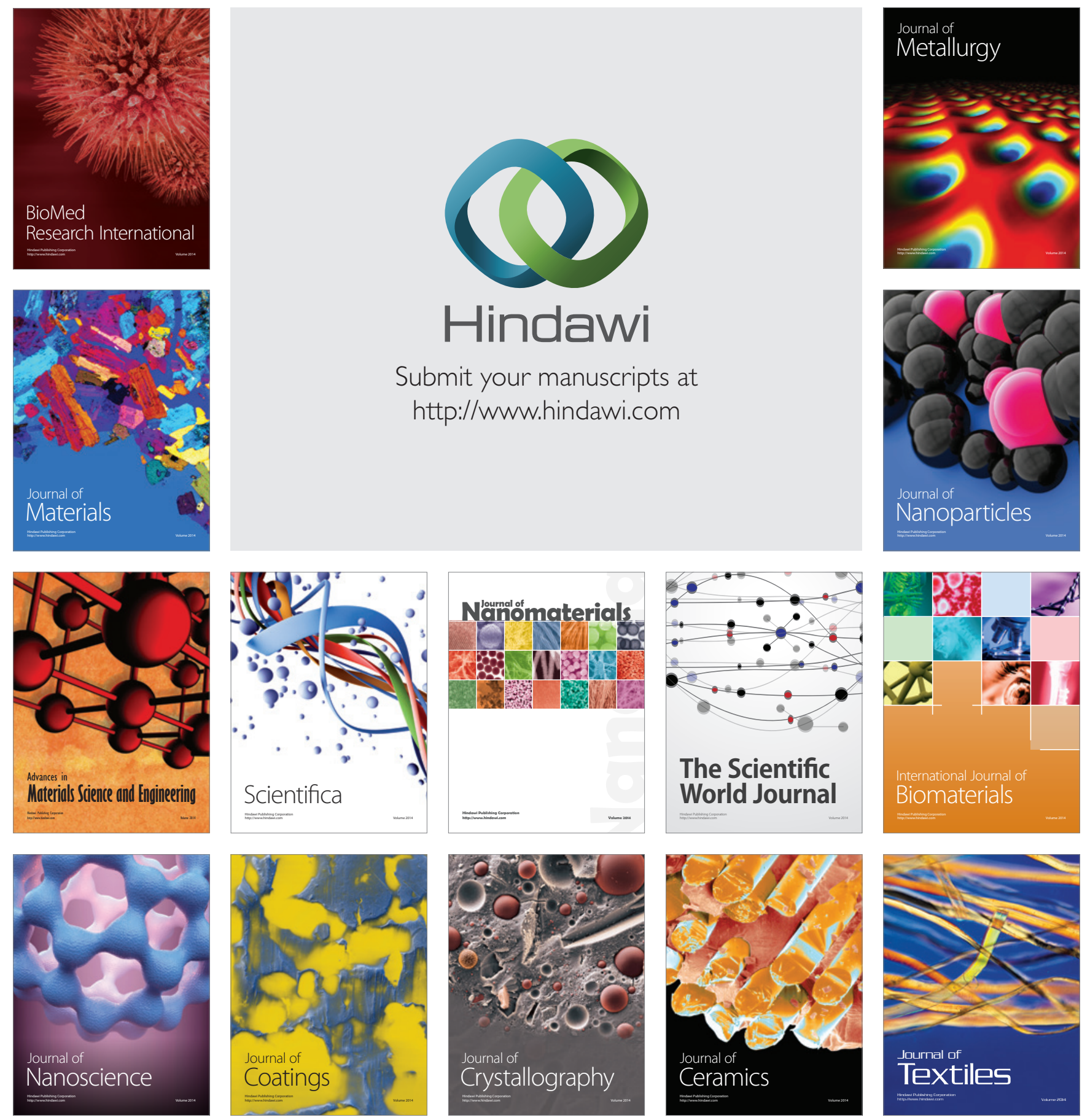